\title{
Volume of tidal gas movement in the nonventilated lung during one-lung ventilation and its relevant factors
}

\author{
Jionglin Wei ${ }^{1+}$, Lei Gao ${ }^{1 \dagger}$, Fafa Sun ${ }^{1}$, Mengting Zhang ${ }^{1}$ and Weidong $\mathrm{Gu}^{1,2^{*}}$
}

\begin{abstract}
Background: The passive ventilation of nonventilated lung results in tidal gas movement (TGM) and thus affects lung collapse. The present study aimed to measure the volume of TGM and to analyse the relevant factors of the TGM index (TGM/body surface area).

Methods: One hundred eight patients scheduled for elective thoracoscopic surgeries were enrolled. Lung isolation was achieved with a double-lumen endobronchial tube (DLT). The paediatric spirometry sensor was connected to the double-lumen connector of the nonventilated lung to measure the volume of TGM during one-lung ventilation (OLV) in the lateral position. The TGM index was calculated. The multiple linear regression was analysed using the TGM index as the dependent variables. Independent variables were also recorded: 1) age, sex, body mass index (BMI); 2) forced vital capacity (FVC), FEV $/$ /FVC, minute ventilation volume (MW); 3) dynamic lung compliance (Cdyn) and peak inspiratory pressure (PIP) during dual lung ventilation; 4) the side of OLV; and 5) whether lung puncture for localization of the pulmonary nodule was performed on the day of surgery. The oxygen concentration in the nonventilated lung was measured at 5 min after OLV, and its correlation with the TGM index was analysed.
\end{abstract}

Results: The volume of TGM in the nonventilated lung during OLV was 78 [37] mL. The TGM index was 45 [20] mL/m² and was negatively correlated with the oxygen concentration in the nonventilated lung at $5 \mathrm{~min}$ after OLV. The multiple linear regression model for the TGM index was deduced as follows: TGM index $\left(\mathrm{mL} / \mathrm{m}^{2}\right)=\mathrm{C}+12.770 \times \mathrm{a}-3.987 \times \mathrm{b}$ $1.237 \times \mathrm{c}-2.664 \times \mathrm{d}$, where $\mathrm{C}$ is a constant $95.621 \mathrm{~mL} / \mathrm{m}^{2}$, a is 1 for males and 0 for females, $\mathrm{b}$ is 1 for right OLV and 0 for left OLV, c is BMI $\left(\mathrm{kg} / \mathrm{m}^{2}\right)$, and dis PIP $\left(\mathrm{cmH}_{2} \mathrm{O}\right)$.

Conclusions: The TGM index is negatively correlated with the oxygen concentration of the nonventilated lung at 5 min after OLV. Sex, side of OLV, BMI and PIP are independently correlated with the TGM index.

Trial registration: This study was registered at ChiCTR (www.chictr.org.cn, ChiCTR1900024220) on July 1, 2019.

Keywords: Tidal gas movement, TGM index, One-lung ventilation, Lung collapse, Double-lumen endobronchial tube, Dynamic lung compliance, Thoracoscopy

\section{Background}

Effective collapse of the nonventilated lung is important for the exposure of critical structures during thoracoscopic surgery [1]. Prior to the thoracic cavity being opened to the atmosphere, positive pressure ventilation of the dependent lung results in mediastinal displacement

\footnotetext{
* Correspondence: hdmz0800@163.com

†ionglin Wei and Lei Gao contributed equally to this work.

'Department of Anaesthesiology, Huadong Hospital, Fudan University, 221

West Yan An road, Jing An District, Shanghai 200040, China

${ }^{2}$ Shanghai Key Laboratory of Clinical Geriatric Medicine, Shanghai, China
}

during one-lung ventilation (OLV) [2]. Subsequently, the transient pressure change in the contralateral hemithorax will cause tidal gas movement (TGM), which is also known as passive ventilation [3-5]. Nitrogen can freely leave from and return to the nonventilated lung during passive ventilation. The presence of nitrogen in the lung serves to delay lung collapse as a consequence of a slower uptake of nitrogen from the lung $[4,6,7]$. By lowering the alveolar partial pressure of oxygen, nitrogen in the lung also results in hypoxic pulmonary vasoconstriction, which may further delay lung collapse [3]. The different volumes

(c) The Author(s). 2020 Open Access This article is distributed under the terms of the Creative Commons Attribution 4.0 International License (http://creativecommons.org/licenses/by/4.0/), which permits unrestricted use, distribution, and 
of TGM may result in different concentrations of oxygen in the nonventilated lung. As a result, TGM affects gas uptake and lung collapse. However, the volume of TGM in the nonventilated lung and its relevant factors remain unknown.

The present study aimed to measure the volume of the tidal gas passively ventilated into the nonventilated lung and to identify the factors associated with the volume of TGM. Furthermore, a mathematical model was established to predict the volume of TGM using multiple linear regression analysis. In addition, the correlation between the volume of TGM and the oxygen concentration in the nonventilated lung at $5 \mathrm{~min}$ after OLV was analysed.

\section{Methods}

After approval of the Ethics Committee of Huadong Hospital affiliated to Fudan University and after obtaining written informed consent, adult patients who were scheduled for elective thoracoscopic surgeries were recruited in our prospective observational study from July 2019 to September 2019. All patients received a lung function test and chest computed tomography (CT) examination preoperatively. Patients with the following conditions were excluded: 1) difficulty in airway management detected by the preoperative assessment, 2) unable to receive double-lumen tube intubation, 3) unable to maintain $\mathrm{SpO}_{2}<90 \%$ during OLV, 4) lung separation failure, and 5) lung bullae or pleural adhesion confirmed by the preoperative CT.

Electrocardiography (ECG), non-invasive blood pressure (NIBP) and pulse oximetry were monitored in all patients before anaesthesia. One hundred percent oxygen was inhaled during the induction of anaesthesia and measurement of the volume of TGM.

All patients received total intravenous anaesthesia [8]. Anaesthesia was induced with propofol $(1-2 \mathrm{mg} / \mathrm{kg})$, sufentanil $(0.3 \mu \mathrm{g} / \mathrm{kg})$, and rocuronium $(1 \mathrm{mg} / \mathrm{kg})$ and was maintained with an infusion of propofol (100$150 \mu \mathrm{g} / \mathrm{kg} \cdot \mathrm{min})$ and remifentanil (0.15-0.2 $\mu \mathrm{g} / \mathrm{kg} \cdot \mathrm{min})$. Intermittent boluses of rocuronium were injected according to a train-of-four neuromuscular monitoring.

The tidal volume was set as $7 \mathrm{~mL} / \mathrm{kg}$ (ideal body weight) during two-lung ventilation and $6 \mathrm{~mL} / \mathrm{kg}$ (ideal body weight) during OLV, respectively. The respiratory rate was $12 / \mathrm{min}$, and the I:E ratio was $1: 2$ without positive end-expiratory pressure (PEEP) $[9,10]$.

Lung isolation was achieved with an appropriately sized left-sided double-lumen endobronchial tube (DLT) [11]. Before placement of the DLT, fibreoptic bronchoscopy examination was performed to inspect the bronchial anatomy and pathology and to remove secretions. The position of the DLT was confirmed under fibre bronchoscopy. Dynamic lung compliance (Cdyn) and peak inspiratory pressure (PIP) during dual-lung ventilation were measured three times, and the average values were calculated for the analysis. The patients were turned to the lateral position when the exhaled oxygen concentration reached $90 \%$. The position of DLT was reconfirmed and adjusted as needed under fibreoptic bronchoscopy $[12,13]$. OLV of the dependent lung was started immediately after the placement of DLT. The fraction of the oxygen concentration in the nonventilated lung was measured at $5 \mathrm{~min}$ after OLV using a gas concentration monitor ( $\mathrm{S} / 5$ Anaesthesia Monitor, GE, Finland). In brief, the nonventilated lung was mechanically ventilated at $5 \mathrm{~min}$ after OLV. End-expiratory oxygen concentration during the first mechanical ventilation was recorded as the fraction of the oxygen concentration in the nonventilated lung at $5 \mathrm{~min}$ after OLV. The accurate location of the DLT was important for lung isolation. If the difference between the actual tidal volumes and the pre-set tidal volumes was more than $10 \mathrm{~mL}$, the patient had to be excluded due to lung separation failure. All procedures were performed by one anaesthesiologist (J.W.).

The volume of TGM in the nonventilated lung was measured in the lateral position using a paediatric spirometry sensor (Pedi-lite Sensor, REF73393, GE, Finland). The sensor was connected to the double-lumen connector of the nonventilated lung [14] which was open to the atmosphere. When the OLV was started, the volume of TGM was consecutively measured three times. The average value was calculated. To increase the comparability between different races, the TGM index (ratio of volume of TGM to body surface area [15]) was calculated and analysed.

The following variables were also recorded: 1) age, sex, body mass index (BMI); 2) forced vital capacity (FVC), FEV1/FVC, minute ventilation volume (MVV) from the pulmonary function test; 3) Cdyn and PIP during dual lung ventilation; 4) side of OLV (left/right); and 5) lung puncture for the localization of the pulmonary nodule on the day of surgery (yes/no).

\section{Statistical analysis}

Data were analysed using the SPSS statistical software package (version 23.0; IBM, Chicago, IL, USA). The TGM index was defined as a continuous outcome variable (dependent variable). Other variables (independent variables) were shown according to the following rules. Continuous variables were analysed with the ShapiroWilk test for normality. Normally distributed variables were expressed as the mean (standard deviation), and nonnormally distributed variables were expressed as the median [interquartile range]. Binomial variables were expressed as numbers (percentages). 
All data were analysed by the single factor correlation analysis. For normally distributed continuous variables, a correlation analysis was performed with Pearson correlation test, while nonnormally distributed data were analysed with Spearman correlation test. Binomial variables were analysed with Student's t-test.

Variables with $P<0.25$ were selected for the subsequent multiple linear regression analysis. Statistical differences were considered to be significant if $P<0.05$.

\section{Sample size estimation}

Sample size was estimated according to the rule-ofthumb of $N \geq 50+8 m$, where $m$ is the number of independent variables [16]. In our pilot study, multiple linear regression analysis showed that there were 4 independent variables. Therefore, the minimum sample size was 82 in the present study.

\section{Results}

One hundred eight patients were enrolled. The volume of TGM was 78 [37] $\mathrm{mL}$. The TGM index was 45 [20] $\mathrm{mL} /$ $\mathrm{m}^{2}$. There was a negative correlation between the TGM index and oxygen concentration in the nonventilated lung at $5 \mathrm{~min}$ after OLV $(r=-0.244, P=0.011)$ (Fig. 1).

There was a significant correlation between the TGM index and sex, BMI, FVC, Cdyn or PIP (Table 1, $P<0.05$ ) (Fig. 2, Fig. 3).

Seven variables with $P<0.25$, including sex, age, BMI, FVC, Cdyn, PIP and side of OLV, were selected to perform the multiple linear regression analysis. Sex, side of OLV, BMI and PIP were independently correlated with the TGM index (Table 2, $P<0.05$ ). The unstandardized

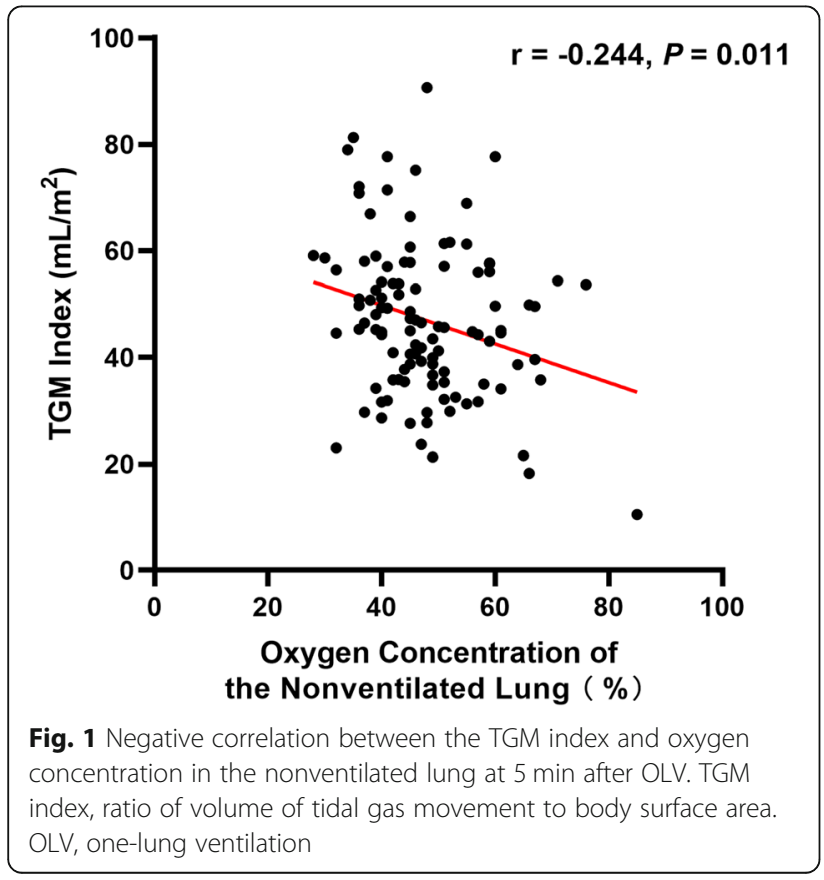

Table 1 Results of single-factor correlation analysis

\begin{tabular}{lll}
\hline & Distribution & $P$ value \\
\hline Age (y), mean (SD) & $56(12)$ & 0.051 \\
Sex (female/male), N & $63 / 45$ & $<0.001$ \\
BMI (kg/m²), median [IQQR] & $24[4]$ & $<0.001$ \\
Pulmonary function tests & & \\
$\quad$ FVC (L), median [IQR] & $2.6[0.9]$ & $<0.001$ \\
$\quad$ MW (L/min), median [IQR] & $29[19]$ & 0.610 \\
FEV ${ }_{1} /$ FVC (\%), median [IQR] & $89[10]$ & 0.752 \\
Pulmonary dynamics during two-lung ventilation & \\
$\quad$ Cdyn (mL/cmH $\left.{ }_{2} \mathrm{O}\right)$, mean (SD) & $48(13)$ & $<0.001$ \\
$\quad$ PIP (cmH $\left.{ }_{2} \mathrm{O}\right)$, median [IQR] & $14.0[2.7]$ & $<0.001$ \\
Side of one-lung ventilation (right/left), N & $39 / 69$ & 0.055 \\
Lung puncture on the surgery day (yes/no), N & $40 / 68$ & 0.659 \\
\hline
\end{tabular}

Continuous data are shown as the mean (SD) or median [IQR] according to normality tests. Binary classification data are shown as $\mathrm{N}$ $S D$ standard deviation; IQR interquartile range; $N$ number $B M I$ body mass index; $B M I$ weight in kilograms divided by height in metres squared; FVC forced vital capacity; MVV minute ventilation volume; FEV1 forced expiratory volume at $1 \mathrm{~s}$; Cdyn dynamic lung compliance; PIP peak inspiratory pressure

coefficients were shown in Table 2. B values were the partial regression coefficients. The constant of the regression model was $95.621 \mathrm{~mL} / \mathrm{m}^{2}$. According to the multiple linear regression analysis, a linear regression model was deduced for the TGM index as follows:

TGM index $\left(\mathrm{mL} / \mathrm{m}^{2}\right)=\mathrm{C}+12.770 \times \mathrm{a}-3.987 \times \mathrm{b}-1.237 \times$ c- $2.664 \times \mathrm{d}$.

C: Constant $=95.621$;

a: male $=1$, female $=0$;

b: right $\mathrm{OLV}=1$, left $\mathrm{OLV}=0$;

c: BMI $\left(\mathrm{kg} / \mathrm{m}^{2}\right)$;

d: PIP $\left(\mathrm{cmH}_{2} \mathrm{O}\right)$.

\section{Discussion}

The present study confirmed that transient changes in the pleural pressure in the nonventilated lung exist during one-lung positive pressure ventilation before opening the thoracic cavity of the nonventilated lung. As a result, it caused tidal movement of gas out of and back into the nonventilated lung. The volume of TGM in the nonventilated lung in the lateral position ranged from 19 to $160 \mathrm{~mL}$.

The measurement of the volume of TGM was performed using a paediatric spirometry sensor. The measurement range was 5 to $300 \mathrm{~mL}$ with an accuracy of $\pm 6 \%$ or $4 \mathrm{~mL}$. It is more accurate to measure small gas movements with a paediatric spirometry senor than with an adult spirometer. Previous studies used different tools to measure the volume of TGM, such as a potentiometer attached to the counter-balance wheel of the spirometer [2], a water-filled spirometer [3], or an ambient pressure oxygen reservoir bag apparatus [4]. Compared to the 

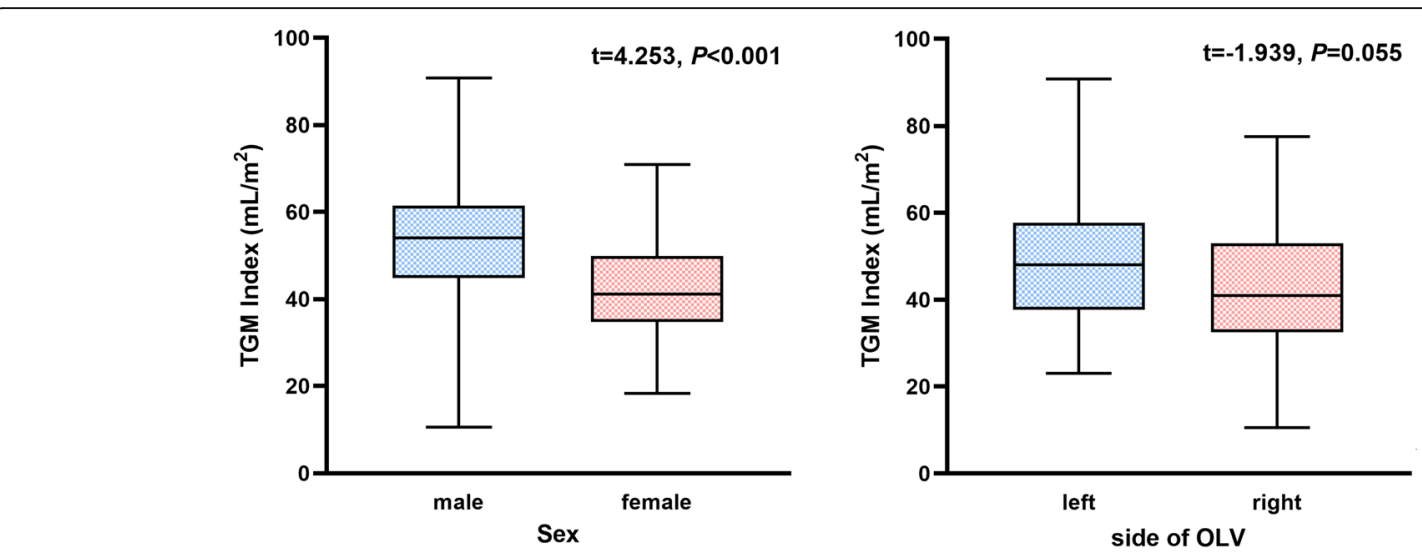

Fig. 2 The correlation between the TGM index and sex or side of OLV, which were two of the binary classification variables, based on multiple linear regression analysis and box plots. The box shows the interquartile range (IQR) and the median of each variable. TGM index, ratio of volume of tidal gas movement to body surface area. OLV, one-lung ventilation

above methods, the paediatric spirometry sensor used in the present study makes the measurement simpler and more accurate. In addition, the volume of TGM can be measured in real-time with a repeatable way by using the paediatric spirometry sensor. The volume of TGM in the present study was smaller than those in other studies $(65-265 \mathrm{~mL})$ [3]. This result may be related to race differences. Therefore, we calculated the TGM index, which allows for comparing TGM between individuals of different sizes.

The oxygen concentration in the nonventilated lung at $5 \mathrm{~min}$ after OLV was negatively correlated with the TGM index in the present study. This could be the result of the nitrogen going into the nonventilated lung by passive ventilation and diluting the oxygen concentration. Since nitrogen is absorbed slower than oxygen, the results suggested that a higher TGM volume may delay lung collapse.
Since there is no research on the analysis of factors associated with the TGM index, ten variables were chosen for the single factor correlation analysis. These variables are the most commonly used clinical indicators and include the patients' general clinical information, lung function variables and basic anaesthesia-related factors. According to the multiple linear regression analysis, sex, side of OLV, BMI and PIP were independently correlated with the TGM index. To the best of our knowledge, this is the first time that a regression model was established to predict the TGM index. Using this model, anaesthesiologists can calculate the TGM index before surgery and evaluate the impact of TGM on the collapse of the nonventilated lung.

In the present study, male patients had a larger TGM index than female patients. Sex was independently correlated with the TGM index. The $B$ value of sex was $12.770 \mathrm{~mL} / \mathrm{m}^{2}$. The underlying reason may be related to
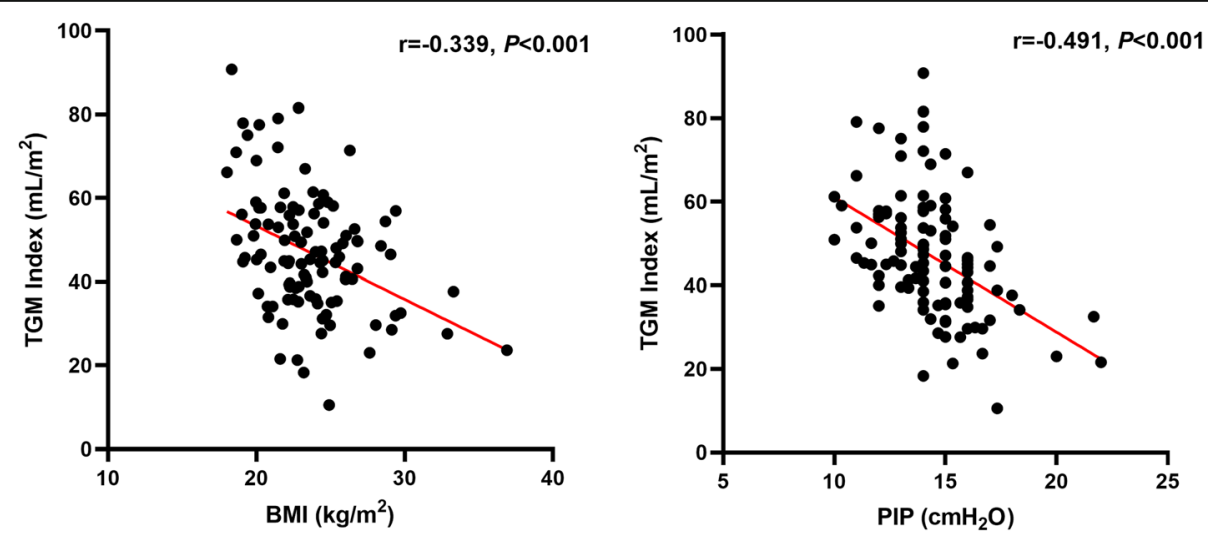

Fig. 3 The correlation between the TGM index and BMI or PIP based on multiple linear regression analysis. TGM index, ratio of volume of tidal gas movement to body surface area. BMI, body mass index. PIP, peak inspiratory pressure 
Table 2 Results of the multiple linear regression analysis

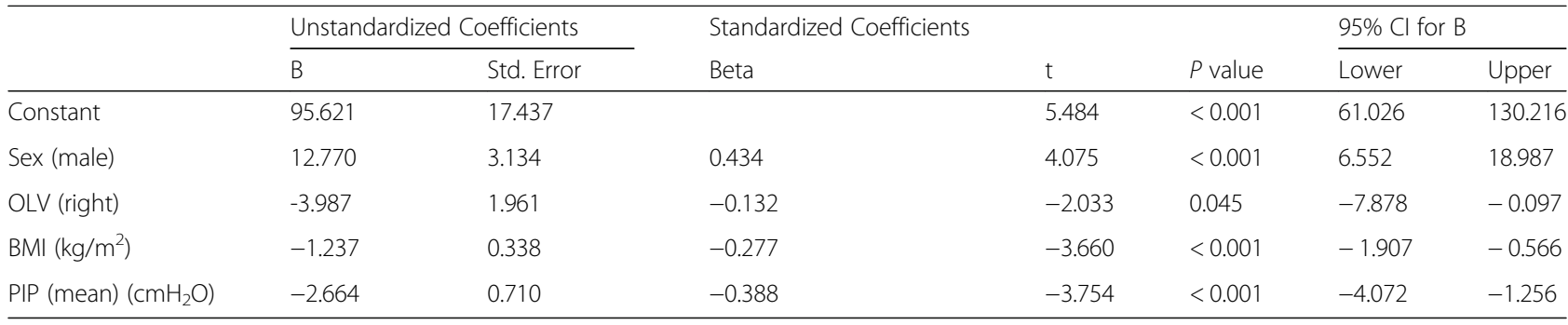

OLV one-lung ventilation; BMI body mass index; PIP peak inspiratory pressure

anatomical differences. The lung volume of adult females is typically $10-12 \%$ smaller than that of males who have the same height and age as females. It results from the differences in the thoracic dimensions between males and females. Because of a greater inclination of ribs, female rib cages could accommodate for a greater volume expansion [17]. During OLV, there is more space for the ventilated lung to expand, less mediastinal movement and less TGM of the nonventilated lung in female patients.

The side of OLV was also independently correlated with the TGM index. Patients with right side OLV produced a lower volume of TGM than those with left side OLV. This may be caused by the anatomical differences between the left and right lungs. The volume of the right lung is larger than that of the left lung, which shares space in the chest with the heart. The right lung has three lobes with 10 segments, while the left lung has only two lobes with 8 segments. Since the tidal volume was the same between left OLV and right OLV, the mediastinal movement was more significant during left OLV than during right OLV. Therefore, the volume of TGM was larger during left OLV than during right OLV.

In the present study, BMI was negatively correlated with the TGM index. The B value is -1.237 . BMI is an important determinant of respiratory function, especially in obese patients [18]. The patients with a higher BMI have low pulmonary/chest wall compliance and increased airway resistance. In addition, there is more adipose tissue in the mediastinum in obese patients. These will consequently make the mediastinal movement more difficult.

PIP depends on airway resistance and pulmonary/chest wall compliance. In the present study, PIP was measured during dual lung ventilation immediately after DLT intubation. The results indicated that the PIP was negatively correlated with the TGM index. The $B$ value was - 2.664. The lung with less PIP was associated with more TGM in the nonventilated lung under the same external force.

There were several limitations in the present study. First, as a single-centre observational study, the sample size was relatively small. Multicentre, large sample observation studies should be performed in the future. Second, TGM was measured only when the tidal volume was set as $6 \mathrm{~mL} / \mathrm{kg}$ of ideal body weight during OLV. Although $6 \mathrm{~mL} / \mathrm{kg}$ of ideal body weight is the commonly used tide volume during OLV, the presented regression model may be unsuitable for other tidal volumes. Third, TGM may indirectly affect oxygenation during surgery by delaying lung collapse. Therefore, the relationship between TGM and oxygenation during surgery needs to be further investigated.

In conclusion, TGM occurs in the nonventilated lung during one-lung ventilation. The TGM index is negatively correlated with the oxygen concentration in the nonventilated lung at $5 \mathrm{~min}$ after OLV. A greater volume of TGM might delay lung collapse during thoracic surgery. Sex, FVC, BMI and PIP are independently correlated with the TGM index. The regression model in the present study may be used to predict the TGM index before surgery and to guide OLV management.

\section{Abbreviations}

BMI: Body mass index; BSA: Body surface area; Cdyn: Dynamic lung compliance; DLT: Double-lumen tube; FEV1: Forced expiratory volume in the first second; FVC: Forced vital capacity; MV: Minute ventilation volume; OLV: One-lung ventilation; PEEP: Positive end-expiratory pressure; PIP: Peak inspiratory pressure; TGM: Tidal gas movement

\section{Acknowledgements}

Not applicable.

\section{Authors' contributions}

JLW and LG were the co-first authors of this article, responsible for the design and implementation of this project, data collection, data statistics, and article writing. FFS contributed to the patient data collection, analysis and interpretation. MTZ contributed to the collection and analysis of the patient data. WDG was the corresponding author of this article in charge of the project, responsible for the expenses, and was involved in the design of the project and modified the manuscript. All authors read and approved the final manuscript.

\section{Funding}

The study was supported by the National Key Research and Development Program of China (No. 2018YFC2002000). Science Foundation of the Science and Technology Commission of Shanghai Municipality (No. 15411965300). Science Foundation of Shanghai Municipal Population and Family Planning Commission (No. 201540067). Foundation of Shanghai Shen Kang Hospital Development Center (No. 16CR3063B). The funding body had no influence of the design of the study and collection, analysis, and interpretation of data and in writing the manuscript. 


\section{Availability of data and materials}

The raw data of the current study are available from the corresponding author on reasonable request.

\section{Ethics approval and consent to participate}

The study was approved by the ethics committee of Huadong Hospital affiliated to Fudan University and the reference number was IRB20180074. This trial was registered at http:/www.chictr.org.cn (ChiCTR1900024220). Written consents to participate were obtained from all participants after enrollment.

\section{Consent for publication}

Not applicable.

\section{Competing interests}

The authors declare that they have no competing interests.

Received: 7 October 2019 Accepted: 13 January 2020

Published online: 22 January 2020

\section{References}

1. Yoshimura T, Ueda K, Kakinuma A, et al. Bronchial blocker lung collapse technique: nitrous oxide for facilitating lung collapse during one-lung ventilation with a bronchial blocker. Anesth Analg. 2014;118(3):666-70

2. Pfitzner J, Peacock MJ, Harris RJ. Speed of collapse of the non-ventilated lung during single-lung ventilation for thoracoscopic surgery: the effect of transient increases in pleural pressure on the venting of gas from the nonventilated lung. Anaesthesia. 2001;56(10):940-6.

3. Pfitzner J, Peacock MJ, McAleer PT. Gas movement in the nonventilated lung at the onset of single-lung ventilation for video-assisted thoracoscopy. Anaesthesia. 1999;54(5):437-43.

4. Pfitzner J, Peacock MJ, Daniels BW. Ambient pressure oxygen reservoir apparatus for use during one-lung anaesthesia. Anaesthesia. 1999;54(5):454-8.

5. Baraka A, Lteif A, Nawfal M, et al. Ambient pressure oxygenation via the nonventilated lung during video-assisted thoracoscopy. Anaesthesia. 2000; 55(6):602-3.

6. Pfitzner J, Peacock MJ, Pfitzner L. Speed of collapse of the non-ventilated lung during one-lung anaesthesia: the effects of the use of nitrous oxide in sheep. Anaesthesia. 2001;56(10):933-9.

7. Pfitzner J. Facilitating lung collapse during one lung ventilation can be rational. Anesth Analg. 2014;119(4):1002-3.

8. Campos $\mathrm{JH}$, Kernstine $\mathrm{KH}$. A comparison of a left-sided broncho-cath ${ }^{\oplus}$ with the torque control blocker univent and the wire-guided blocker. Anesth Analg. 2003;96(1):283-9.

9. Brassard CL, Lohser J, Donati F, et al. Step-by-step clinical management of one-lung ventilation: continuing professional development. Can J Anesth. 2014:61(12):1103-21.

10. Şentürk M. New concepts of the management of one-lung ventilation. Anesthesiology. 2006:19(1):1-4

11. Pedoto A. How to choose the double-lumen tube size and side: the eternal debate. Anesthesiol Clin. 2012;30(4):671-81.

12. Bahk JH, Oh YS. A new and simple maneuver to position the left-sided double-lumen tube without the aid of fiberoptic bronchoscopy. Anesth Analg. 1998;86(6):1271-5

13. Suzuki A, Tanaka H, Tampo A, et al. A simple alternative method to confirm lung separation. Anesth Analg. 2012;114(3):701-2.

14. Schmide J, Irouschek A, Heinrich S, et al. Another sensitive and safe method to observe lung separation during single-lung ventilation. Anesth Analg. 2013;116(2):509-10.

15. Mosteller RD. Simplified calculation of body surface area. N Engl J Med. 1987;317(17):1098

16. Green SB. How many subjects does it take to do a regression analysis. Multivar Behav Res. 1991;26(3):499-510.

17. Bellemare F, Jeanneret A, Couture J. Sex differences in thoracic dimensions and configuration. Am J Respir Crit Care Med. 2003;168(3):305-12.

18. Pelosi $\mathrm{P}$, Croci M, Raagnan I, et al. The effects of body mass on lung volumes, respiratory mechanics, and gas exchange during general anesthesia. Anesth Analg. 1998;87(3):654-60

\section{Publisher's Note}

Springer Nature remains neutral with regard to jurisdictional claims in published maps and institutional affiliations.

Ready to submit your research? Choose BMC and benefit from:

- fast, convenient online submission

- thorough peer review by experienced researchers in your field

- rapid publication on acceptance

- support for research data, including large and complex data types

- gold Open Access which fosters wider collaboration and increased citations

- maximum visibility for your research: over $100 \mathrm{M}$ website views per year

At $\mathrm{BMC}$, research is always in progress.

Learn more biomedcentral.com/submissions 